\title{
NESSI OSS Workshop
}

\author{
The NESSI Open Source Working Group \\ http://www.nessi-europe.eu
}

\section{Introduction}

The NESSI Technology Platform aims to provide a unified view for European research and development in Services Architectures and Software Infrastructures that will define technologies for new, open, industrial solutions and societal applications that enhance the safety, security and well-being of citizens.

Open Source nourishes and supports the dynamic of NESSI's vision. Open source has a catalytic effect by enabling acceleration of changes that are already under way in the ICT economy.

Open Source increases competition, accelerate open standards adoption and evolution, provides a working model addressing erosion of geographic boundaries, support the services economy by increasing transparency while positioning software as a public good and optimizing costs.

The NESSI OSS Working Group can be seen as transversal support to achieve the NESSI vision by creating the appropriate conditions and environment that will facilitate adoption of Open Source technologies, collaborative development process and synergies with Open Source proponents.

NESSI is seeking to identify what are the orientations and the new perspectives given by OSS to tackle the challenges emerging in the coming years.

NESSI has defined NEXOF, a coherent open service framework designed to guarantee quality of service, ubiquitous operation and continuous availability to enable delivery of large scale service applications based on open standard interfaces. In the strategy and plan to build such a framework, NESSI promotes openness and is dedicated to building solutions that can be used by anybody.

The NESSI Open Source WG promotes integration of Open Source within NESSI platforms and services and supports Open Source development communities wishing to participate and collaborate with NESSI.

Software landscape is increasingly interconnected. The evolution to SOA makes it easier for software developments to be decomposed and distributed. An Open Source approach makes sense in domains where interoperability and software sharing enables the development of business for large scale service applications.

Open source is a driving force for the expansion of the European software industry, but its progress has been inhibited by factors such as intellectual property issues, lack of OSS in high education and the fragmentation of the open-source community.

The NESSI OSS WG encourages greater use of OSS in education to support the update of the workforce skills, address legal issues and promote business models to support adoption of NESSI Open Framework and OSS at large, federates open source efforts according to interest groups.

The workshop will propose and discuss concerted approaches to address some of these challenges. In this perspective, a focus will be put on open source in high education and the need for open services platforms. 


\section{Workshop Objectives}

- Raise awareness about NESSI OSS activities

- Facilitate sharing and coordination between academia initiatives for teaching OSS

- Get feedback on collaboration schema and promote Interest groups for Open Source development communities wishing to participate and collaborate with NESSI

- Get feedback on what the obstacles are for building an open service infrastructure and what technologies or solutions might help.

\section{Format}

- NESSI and NESSI OSS Overview presentation

- Interactive workshop session on Coordinated approach for OSS education

- challenges to overcome according to existing experience

- OSS skills certification programme

- proposals for curricular content: topics for IT courses, how to federate efforts?

- Interactive workshop session on Building the Open Source Service Platform

- Collaboration schema presentation

- NEXOF open construction cycles

- Feedback from the interest groups on current activities and call for participation

- Discussion and questions from the audience

\section{Target Participants}

- NESSI OSS members

- OSS communities, representatives of OSS projects

- Representatives of universities with OSS curricula

- R\&D projects representatives

More information: http://www.nessi-europe.eu 